\title{
Titinopathy in a Canadian Family Sharing the British Founder Haplotype
}

\author{
Gerald Pfeffer, Jeffrey T. Joseph, A. Micheil Innes, J. Bevan Frizzell, Ian J. Wilson, \\ A. Keith W. Brownell, Patrick F. Chinnery
}

Can J Neurol Sci. 2014; 41: 90-94

Hereditary myopathy with early respiratory failure (HMERF, OMIM 603689) is an autosomal dominant myopathy, often characterised by respiratory muscle weakness early in the disease course. This condition shares many similarities with myofibrillar myopathy (MFM) and it has been suggested that HMERF be considered within this disease category ${ }^{1,2}$. It is caused by mutations in TTN, which encodes the giant sarcomeric protein titin. The hotspot for mutations causing this disorder appears to be in the 119th fibronectin 3 (FN3) domain in the titin A-band, with the p.C30071R mutation ${ }^{3,4}$ being most common (using Genebank\#: AJ277892 as the reference sequence), although other mutations have recently been described ${ }^{1,2,5,6}$. A mutation in the kinase domain of TTN (p.R32450W) was associated with this condition ${ }^{7}$, but this variant is also designated as a low-frequency polymorphism (rs140319117) in the European population.

Two studies have now revealed that TTN mutations are responsible for about $5 \%$ of cases of undiagnosed $\mathrm{MFM}^{1,5}$, and the disease is now demonstrated to be internationally distributed $^{2,5,6}$, beyond the British/Finnish founder effect which was initially described ${ }^{3,4}$. To date, only a single family with this disease has been reported from Canada ${ }^{5}$. We report the second Canadian family with this disease, caused by the common p.C30071R mutation in TTN, and demonstrate that this patient shares a founder haplotype with previously reported families from the North East of England.

\section{METHODS}

\section{Case report}

Clinical records and diagnostic results were reviewed from the proband, his affected father, and paternal grandfather. The proband provided consent for publication of anonymised clinical data.

\section{Muscle magnetic resonance imaging}

Magnetic resonance imaging (MRI) of the pelvis and both lower limbs (hips to ankles) was performed using a 1.5T Discovery 450 (General Electric Medical Systems). Axial T1 (TR 530, TE 12) and T2 fat sat (TR 5471, TE 88) sequences were acquired through the lower limbs using the body coil, and axial T1 (TR 509, TE 14) and STIR (TR 4625, TE 54) sequences acquired through the pelvis using the 8 channel cardiac coil. A single rater visually quantified the degree of muscle atrophy in individual muscles using the following scale: $0=$ no increased intramuscular fat, 1 = fat less extensive than muscle, $2=$ fat equal to muscle, $3=$ fat more extensive than muscle, and $4=$ complete fatty replacement.

\section{Muscle pathology}

Snap-frozen muscle biopsies received standard tissue staining, including hematoxylin-eosin and Gomori trichrome stains. Immunoperoxidase staining was performed on formalinfixed paraffin-embedded tissue using the Leica Bond Polymer Refine Detection kit on a Leica Bond III platform. Antibodies used included desmin (Dako clone D33 at 1/400 dilution) and TDP-43 (Protein Tech rabbit polyclonal at 1/4000). Electron microscopy was prepared by fixation in paraformaldehyde, embedding in epoxy resin, and visualized in thick sections stained with toluidine blue or by electron microscopy on a Hitachi electron microscope using osmium tetroxide fixation and uranyl acetate staining.

\section{Genetic testing}

Sanger sequencing was performed for the two previously described TTN mutations at positions p.30071 and p.32450 (as previously described ${ }^{1}$ ). Polymerase chain reaction was performed with Immolase (Bioline) according to manufacturer's protocol, using $\sim 50 \mathrm{ng}$ of DNA, $0.25 \mathrm{mM}$ of each oligonucleotide and $4 \mathrm{mM} \mathrm{MgC}$, for 30 cycles with annealing temperature of $60-65^{\circ} \mathrm{C}$. Sequencing was performed using BigDye, (Applied Biosystems) according to the manufacturer's protocol with an ABI 3130XL sequencer. Additional single nucleotide variants flanking the disease mutation which defined the shared haplotype as described were Sanger sequenced to compare this family with those we have described previously. Analysis of the shared haplotype was performed as published ${ }^{1}$.

\section{RESULTS}

\section{Case report}

The family pedigree is outlined in Figure 1. The proband (III:2) is a 29-year-old man who was first investigated at the age

From the Institute of Genetic Medicine (GP, IJW, PFC), Newcastle University, Newcastle, United Kingdom; Department of Pathology and Laboratory Medicine (JTJ), Department of Medical Genetics (AMI), Department of Radiology (JBF), Department of Clinical Neurosciences (AKWB), University of Calgary, Calgary, Alberta, Canada. Received July 3, 2013. Final Revisions Submitted August 28, 2013. Correspondence to: Patrick F. Chinnery, Institute of Genetic Medicine, Central Parkway, Newcastle, NE1 3BZ, United Kingdom. Email: patrick.chinnery@ncl.ac.uk. 


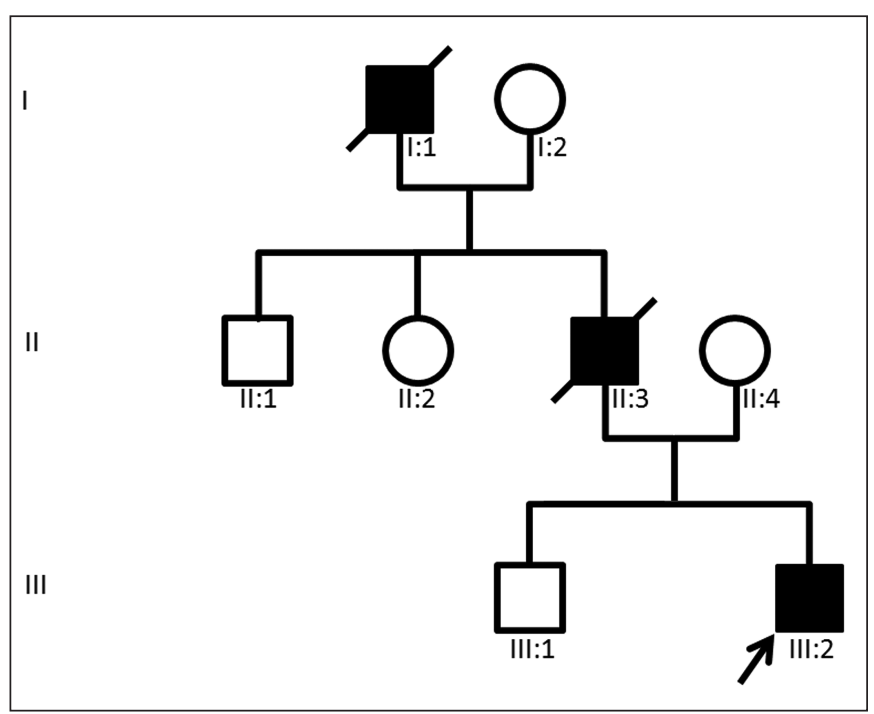

Figure 1: The pedigree structure indicates autosomal dominant inheritance. Patients II:3 and I:1 are respectively the father and grandfather of the proband, and died from complications of neuromuscular respiratory failure.

of five years because of a history of muscle disease in his father (II:3). At the time neurological examination was normal. He was reassessed at age 16 and although still asymptomatic, he had mild ankle dorsiflexor and great toe flexor weakness bilaterally. Creatine kinase $(\mathrm{CK})$ was elevated at $360 \mathrm{u} / \mathrm{L}$ (reference range 0195). His most recent examination at age 30 demonstrated $4 / 5$ weakness in ankle dorsiflexion, inversion and eversion. He had ankle plantarflexion contractures and was unable to walk on his heels. Otherwise he had no functional limitations. His father had developed distal lower extremity weakness at age 25 which gradually generalised. He was wheelchair-bound and required BiPAP for 18 months before he died of respiratory failure at age 36. His paternal grandfather (who originated from England) had onset of distal lower extremity weakness in his 30's which gradually worsened to include proximal and distal upper and lower extremities, and neck flexors. In his last years of life he was wheelchair-bound, required bilevel positive airway pressure (BiPAP) and continuous positive airway pressure (CPAP), and died in his early 60's of respiratory failure.

\section{Muscle MRI}

Fatty infiltration was most pronounced in the semitendinosus muscles and anterior compartment muscles in the lower leg (Figure 2). Other muscles which were markedly affected included obturator externus, pectineus, and tibialis posterior. Detailed reporting of individual muscles with visual quantitation of fatty infiltration is presented in the Table.

\section{Muscle pathology}

The muscle biopsy performed in 1988 on Patient II:3 (the proband's father) revealed a severe, chronic myopathy having significant variation in myofibre size, including many tiny fibres, increased connective tissue, internalized nuclei, and fatty infiltration (Figure 3). Some myofibres contained small vacuoles, while others contained multiple cytoplasmic bodies. Scattered fibres had large inclusions filled with debris. Immunoperoxidase stains demonstrated occasional dense deposits of desmin. Frequent small myofibres showed sarcoplasmic immunoreactivity for TDP-43.

In thick plastic sections stained with toluidine blue, degenerate myofibres contained autophagic vacuoles, cytoplasmic bodies, and dense, irregular deposits (Figure 4). Ultrastructurally, the earliest change was disruption of the sarcomeric pattern with some Z-band streaming. Extensively affected fibres contained large autophagic vacuoles and condensed aggregates of thick filaments, or were highly atrophic and contained frequent cytoplasmic bodies.

\section{Genetic testing}

Sanger sequencing of leucocyte DNA from the proband identified the heterozygous g.274375T $>\mathrm{C}$ mutation (predicted to cause a p.C30071R substitution). Sequencing of the TTN kinase domain revealed wild-type sequence. Sequencing of polymorphisms surrounding the mutation demonstrated a
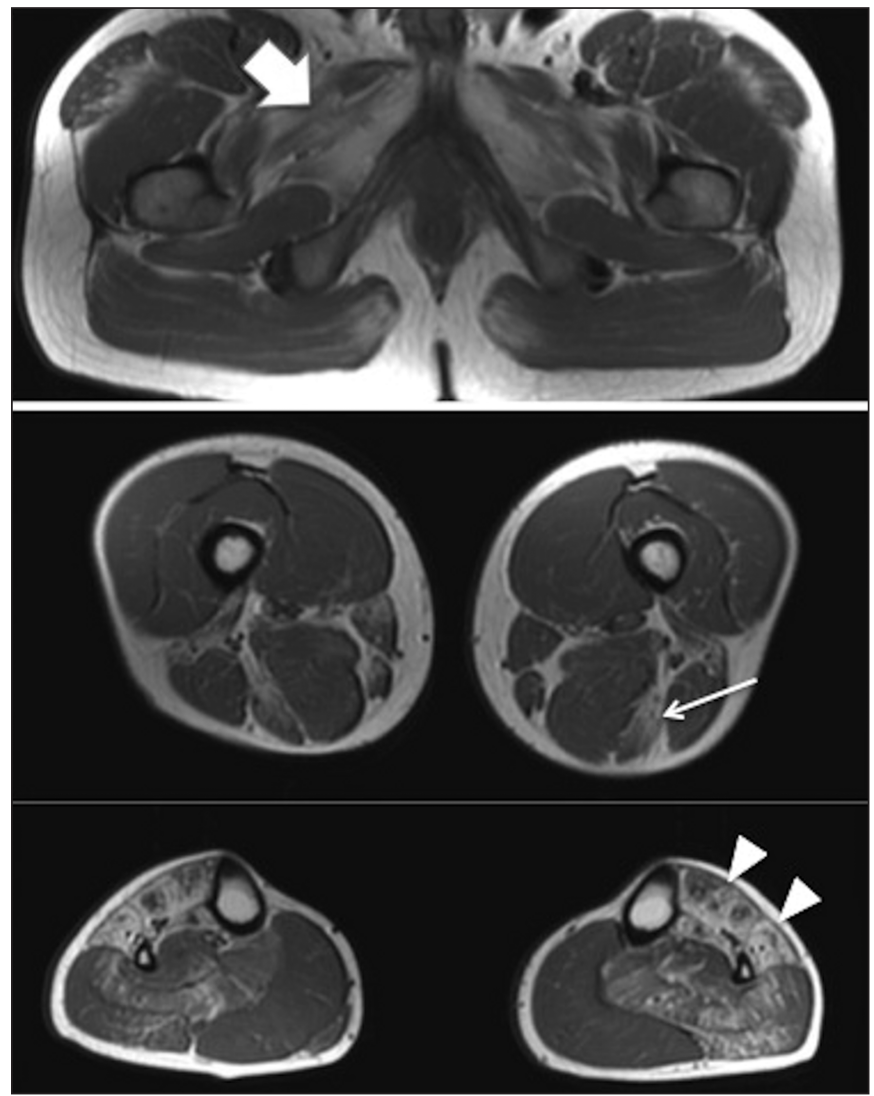

Figure 2: Representative axial T1-weighted MRI images through the lower hips, mid-thigh, and upper calves highlighting the pattern of muscle atrophy in the proband. Thick arrow indicates obturator externus and pectineus. Thin arrow indicates semitendinosus. Arrowheads indicate anterior and peroneal compartments of calf (including tibialis anterior, extensor digitorum, peroneus longus and brevis) 
Table: Visual quantification of muscle atrophy on muscle MRI

\begin{tabular}{l|l|l|l}
\hline Region & Muscle & Score & Comment \\
\hline \multirow{5}{*}{ Pelvis } & Gluteus maximus & 3 & Symmetric, medial \\
\cline { 2 - 4 } & Gluteus medius/minimus & 2 & Symmetric, lateral \\
\cline { 2 - 4 } & Psoas & 2 & $\mathrm{R}>\mathrm{L}$ \\
\cline { 2 - 4 } & Iliacus & 3 & $\mathrm{R}>\mathrm{L}$ \\
\hline \multirow{4}{*}{ Upper leg } & Pectineus/obturator externus & 3 & Symmetric \\
\cline { 2 - 4 } & Rectus femoris & 1 & Symmetric \\
\cline { 2 - 4 } & Semitendinosus & $3-4$ & Symmetric \\
\cline { 2 - 4 } & Biceps femoris (short head) & 2 & Symmetric \\
\cline { 2 - 4 } & Gracilis & 2 & $\mathrm{R}>\mathrm{L}$ \\
\cline { 2 - 4 } & Sartorius & $2(\mathrm{R}), 1(\mathrm{~L})$ & $\mathrm{R}>\mathrm{L}$ \\
\cline { 2 - 4 } & Adductor longus & 1 & $\mathrm{~L}>\mathrm{R}$ \\
\cline { 2 - 4 } & Peroneal/anterior compartment & $3-4$ & Symmetric \\
\cline { 2 - 4 } & Tibialis posterior & 4 & Symmetric \\
\cline { 2 - 4 } & Flexor digitorum longus/flexor hallucis longus & $1-2$ & Symmetric \\
\cline { 2 - 4 } & Soleus/gastrocnemus (lateral head) & 2 & $\mathrm{~L}>\mathrm{R}$ \\
\hline
\end{tabular}

$\mathrm{R}=$ right; $\mathrm{L}=$ left. Scoring for muscle atrophy as follows: $0=$ no increased intramuscular fat, $1=$ fat less extensive than muscle, $2=$ fat equal to muscle, 3 = fat more extensive than muscle, and $4=$ complete fatty replacement

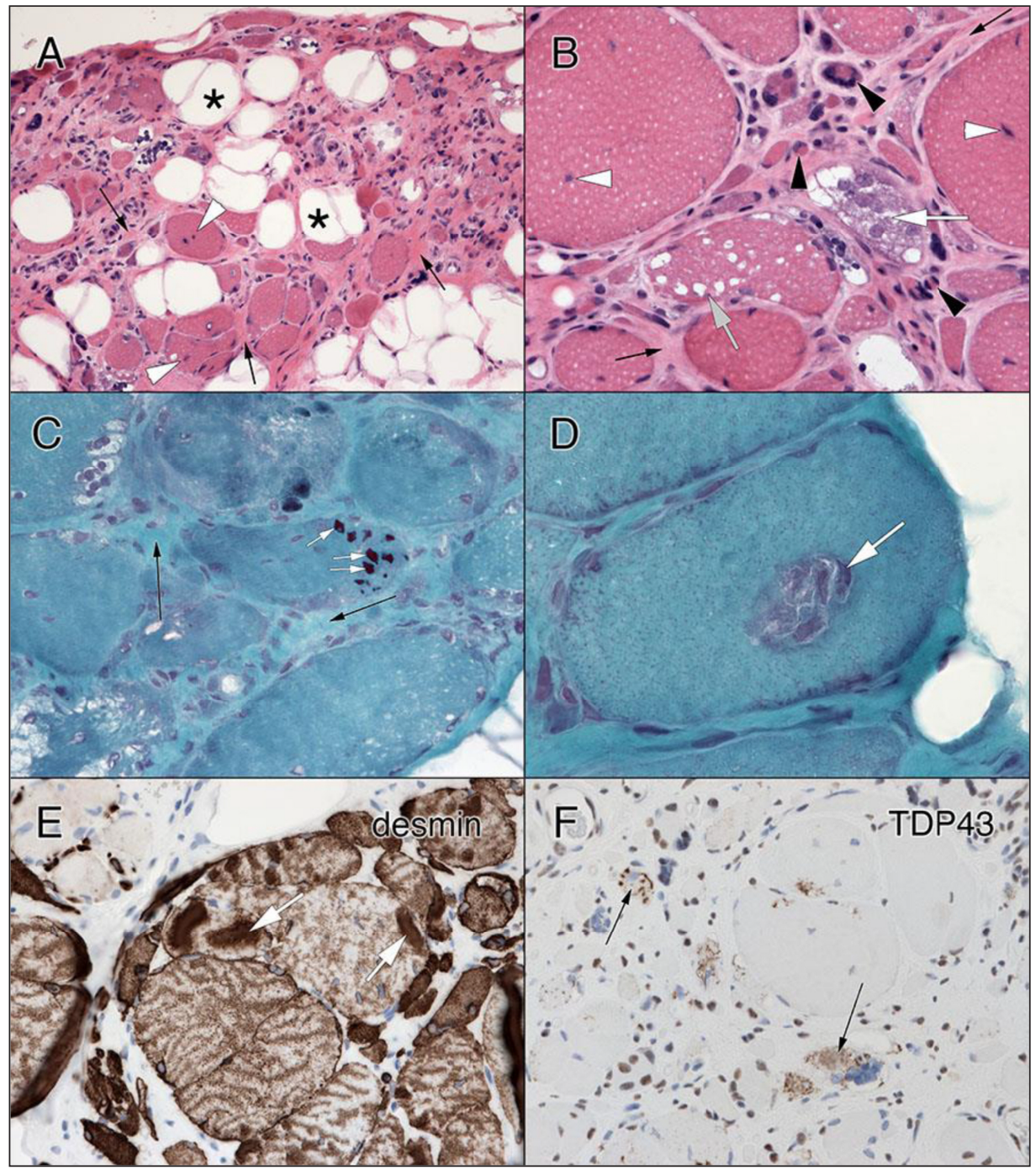

Figure 3: Microscopy of muscle biopsy from Patient II:3. The biopsy demonstrated a severe, chronic myopathy, including fatty infiltration (asterisks), increased endomysial connective tissue (black arrows), increased internalized nuclei (white arrowheads), and many tiny myofibres (A). High magnification demonstrates these same findings, as well as vacuolated myofibre (gray arrow) and myofibres undergoing myophagocytosis (white arrow) (B). The Gomori trichrome shows a fibre containing multiple cytoplasmic bodies (white arrows) and the increased connective tissue (black arrows) (C). Occasional fibres had a large vacuole containing basophilic material (white arrow) (D). Some fibres contained aggregates of desmin (white arrows) (E), while scattered small fibres had multiple TDP43-immunoreactive aggregates (black arrows) $(F)$. A and B: Hematoxylin-eosin stain. $C$ and $D$ : Gomori trichrome stain. E: Desmin immunoperoxidase stain on formalin fixed tissue. F: TDP43 immunoperoxidase stain on formalin fixed tissue. 


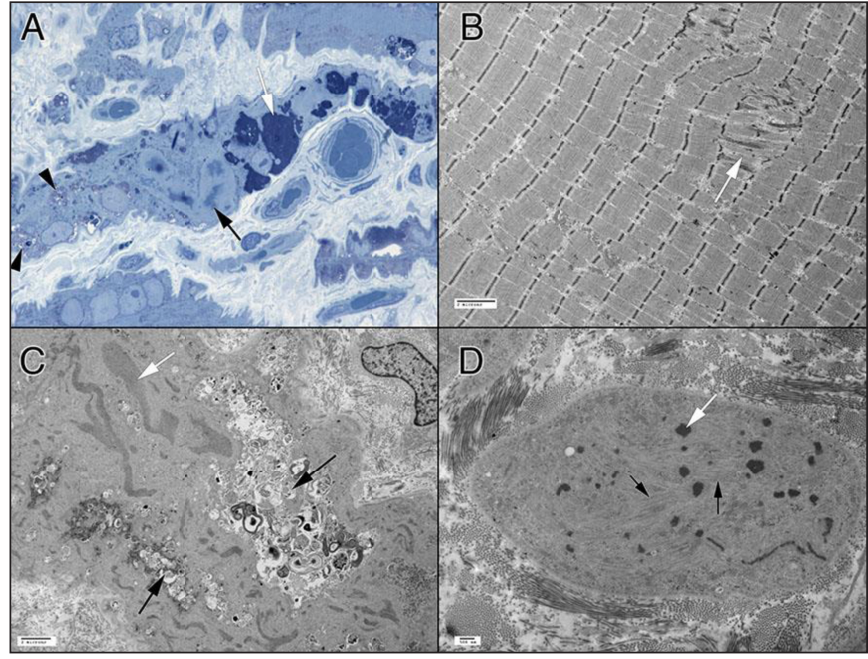

common haplotype with our previously described families ${ }^{1}$, and is represented in Figure 5. The polymorphisms rs6706354, rs6706483, and rs919177 are present in all previously reported patients who have had this analysis, and are also present in the proband from the current report. Phased haplotype analysis indicates that the larger 789 kilo-base pair (kbp) haplotype has not been previously reported ${ }^{8}$, suggesting that the haplotype is unique to the $\mathrm{g} .274375 \mathrm{~T}>\mathrm{C}$ mutation.

\section{Discussion}

We present the second Canadian family with HMERF caused by the p.C30071R mutation, and the first family outside of England confirmed to share the previously described founder
Figure 4: Toluidine-stained plastic section (A) and ultrastructure of muscle from Patient II:3. The degenerate myofibre in the plastic section is embedded in a collagenous matrix and contains irregular, densely staining aggregates (white arrow), more lightly staining and uniform cytoplasmic bodies (black arrow), and autophagic vacuoles (black arrowheads) (A). The earliest ultrastructural change was streaming of myofibrils (white arrow), which disrupted the normal sarcomeric structure and created dense aggregates perpendicular to the Z-line orientation $(B)$. More degenerate myofibres contained autophagic vacuoles (black arrows) and aggregates of lightly osmiophilic thick filaments embedded in pale thin filaments (white arrow) (C). Highly atrophic fibres contained frequent cytoplasmic bodies (white arrow) and streams of haphazardly arranged filaments (black arrows) (D).

haplotype. This provides further evidence indicating that this disease is more common than previously believed, and that the prevalence described in the $\mathrm{UK}^{1}$ (relating to the founder effect) may not be an overrepresentation of the disease prevalence internationally. Our patient is presumably related to one of the prior UK families but detailed reviews of family records for several generations could not identify a shared relative. This is not surprising because the very small size of the shared UK haplotype $(172 \mathrm{kbp})$ indicates that this is an ancient mutation ${ }^{1}$ that is likely scattered within the UK population, and with this report has been confirmed to be present in at least one country outside the UK. It is possible that the UK haplotype is present in the other reported patients with the p.C30071R mutation, and if true suggests that all patients with this mutation are related (i.e: the mutation occurred only once from a single common founder). Using the haplotype data presented in this work, this question could be investigated in the course of future study.

The MRI and muscle pathology investigations reinforce the position taken by prior reports, that HMERF is a subtype of MFM $^{1,2}$. In our patient, muscle MRI demonstrated fatty

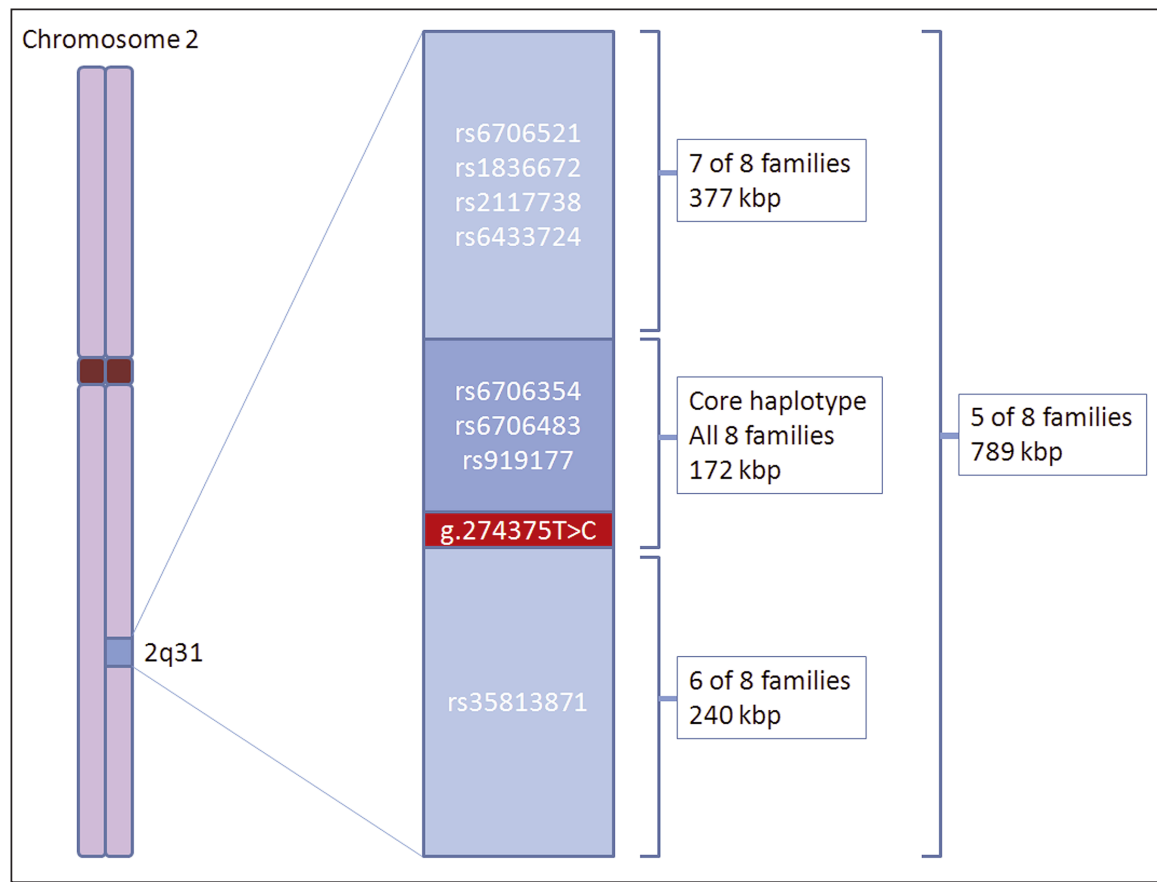

Figure 5: Schematic diagram of manually constructed haplotype (assuming the minimum number of recombinations) indicate that our patient shares a haplotype of at least $172 \mathrm{kbp}$ with 8 other HMERF families with the p.C30071R TTN mutation (Families F1-F5 in reference 1 and $A-C$ in reference 4$)$. He shares a larger haplotype of $789 \mathrm{kbp}$ with 5 of these families (Families A, B, F2, F3, F4). (kbp=kilobase pairs; g.274375T $>C$ is the genomic coordinate for the p.C30071R mutation using Genebank\#: AJ277892 as the reference sequence) 
infiltration of various muscles, particularly the obturator externus, semitendinosus, and peroneal muscles. These are the most commonly affected muscles in $\mathrm{HMERF}^{4}$, but are also within a radiologic differential diagnosis for $\mathrm{MFM}^{9}$. The pathological findings in the father's muscle biopsy were similar to those described in $\mathrm{HMERF}^{3,5}$, including disruption of sarcomeric structure by Z-disk dispersion, formation of cytoplasmic bodies, vacuoles containing degenerate debris, and condensed aggregates of different proteins. These pathological changes are also diagnostic features of MFM. It has been suggested, to avoid confusion and to improve our ability to recognise this condition, that HMERF be designated as MFMtitinopathy ${ }^{1}$.

In conclusion, patients with muscle disease having onset in adulthood, with distal leg weakness and/or respiratory muscle weakness, and autosomal dominant inheritance, should have titinopathy considered in the differential diagnosis. Creatine kinase may be normal but more often is modestly elevated (up to $1000 \mathrm{u} / \mathrm{L})^{4}$. Muscle MRI abnormalities invariably include fatty infiltration of the semitendinosus and anterior compartment of the lower leg muscles ${ }^{4,10}$. Muscle pathology shows features of $\operatorname{MFM}^{(1-3)}$, but may also be nonspecific ${ }^{1,4}$. Sequencing of the 119th FN3 domain of TTN for this single exon should be included in the investigations for the differential diagnosis of MFM.

\section{ACKNOWLEDGEMENTS}

The authors would like to acknowledge the participation of the proband and his family, as well as the assistance of Linda MacLaren. GP is the recipient of a Bisby fellowship from the Canadian Institutes of Health Research. PFC is an Honorary Consultant Neurologist at Newcastle upon Tyne Foundation Hospitals NHS Trust, is a Wellcome Trust Senior Fellow in Clinical Science (084980/Z/08/Z), and a UK NIHR Senior Investigator. PFC receives additional support from the Wellcome Trust Centre for Mitochondrial Research (096919Z/11/Z), the Medical Research Council (UK) Centre for Translational Research in Neuromuscular Diseases, and EU FP7 TIRCON, and the National Institute for Health Research (NIHR) Newcastle Biomedical Research Centre based at Newcastle upon Tyne Hospitals NHS Foundation Trust and Newcastle University.

\section{REFERENCES}

1. Pfeffer G, Barresi R, Wilson IJ, et al. Titin founder mutation is a common cause of myofibrillar myopathy with early respiratory failure. J Neurol Neurosurg Psychiatry. 2013 Mar 13. [Epub ahead of print]

2. Izumi R, Niihori T, Aoki Y, et al. Exome sequencing identifies a novel TTN mutation in a family with hereditary myopathy with early respiratory failure. J Hum Genet. 2013 May;58(5):259-66.

3. Ohlsson M, Hedberg C, Bradvik B, et al. Hereditary myopathy with early respiratory failure associated with a mutation in A-band titin. Brain. 2012 Jun;135(Pt 6):1682-94.

4. Pfeffer G, Elliott HR, Griffin H, et al. Titin mutation segregates with hereditary myopathy with early respiratory failure. Brain. 2012 Jun;135(Pt 6):1695-713.

5. Toro C, Olive M, Dalakas MC, et al. Exome sequencing identifies titin mutations causing hereditary myopathy with early respiratory failure (HMERF) in families of diverse ethnic origins. BMC Neurol. 2013 Mar 20;13:29.

6. Palmio J, Evila A, Chapon F, et al. Hereditary myopathy with early respiratory failure: occurrence in various populations. J Neurol Neurosurg Psychiatry. 2013 Apr 19. [Epub ahead of print].

7. Lange S, Xiang F, Yakovenko A, et al. The kinase domain of titin controls muscle gene expression and protein turnover. Science. 2005 Jun 10;308(5728):1599-603.

8. 1000 Genomes Project Consortium, Abecasis GR, Auton A, Brooks LD, DePristo MA, Durbin RM, et al. An integrated map of genetic variation from 1,092 human genomes. Nature. 2012 Nov 1;491(7422):56-65.

9. Wattjes MP, Kley RA, Fischer D. Neuromuscular imaging in inherited muscle diseases. Eur Radiol. 2010 Oct;20(10):2447-60.

10. Birchall D, von der Hagen M, Bates D, Bushby KM, Chinnery PF. Subclinical semitendinosus and obturator externus involvement defines an autosomal dominant myopathy with early respiratory failure. Neuromuscul Disord. 2005 Oct;15(9-10):595-600. 